\title{
About a New Kind of Ramanujan-Type Series
}

\author{
Jesús Guillera
}

\section{CONTENTS}

1. Introduction

2. Ramanujan-Type Formulas

3. Supporting the Conjecture

4. Related Formulas

References
2000 AMS Subject Classification: Primary 33C20

Keywords: Ramanujan-type series for Pi, generalized hypergeometric series
We propose a new kind of Ramanujan-type formula for $1 / \pi^{2}$ and conjecture that it is related to the theory of modular functions.

\section{INTRODUCTION}

In my papers [Guillera 02, Guillera 03], I prove the identities

$$
\begin{aligned}
& \sum_{n=0}^{\infty} \frac{(-1)^{n}\left(\frac{1}{2}\right)_{n}^{5}}{n !^{5} 2^{10 n}}\left(820 n^{2}+180 n+13\right)=\frac{128}{\pi^{2}} \\
& \sum_{n=0}^{\infty} \frac{\left(\frac{1}{2}\right)_{n}^{3}\left(\frac{1}{4}\right)_{n}\left(\frac{3}{4}\right)_{n}}{n !^{5} 2^{4 n}}\left(120 n^{2}+34 n+3\right)=\frac{32}{\pi^{2}} \\
& \sum_{n=0}^{\infty} \frac{(-1)^{n}\left(\frac{1}{2}\right)_{n}^{5}}{n !^{5} 2^{2 n}}\left(20 n^{2}+8 n+1\right)=\frac{8}{\pi^{2}} .
\end{aligned}
$$

Inspired by these results and by Ramanujan's formulas [Borwein and Borwein 87, Chudnovsky and Chudnovsky 88, Ramanujan 14], I had the feeling that more formulas of the same type could exist. So, I experimented in order to find them. I now describe that research.

\section{RAMANUJAN-TYPE FORMULAS}

The kind of formulas we are looking for have the form

$$
\sum_{n=0}^{\infty} \frac{B(n)}{q^{n}}\left(a n^{2}+b n+c\right)=\frac{d \sqrt{k}}{\pi^{2}}
$$

where $d, k, a, b, c$ are integers, $B(n)=n !^{-5} C(n)$ or $B(n)=(-1)^{n} n !^{-5} C(n)$, and $C(n)$ is the product of 5 rising factorials of fractions smaller than unity satisfying the following condition: For every denominator in the fraction of a rising factorial, we must have rising factorials with all possible nonreducible fractions corresponding to that denominator. Taking this into account, we have the following cases for $C(n)$ :

$$
\left(\frac{1}{2}\right)_{n}\left(\frac{1}{2}\right)_{n}\left(\frac{1}{2}\right)_{n}\left(\frac{1}{2}\right)_{n}\left(\frac{1}{2}\right)_{n},
$$

(c) A K Peters, Ltd $1058-6458 / 2003 \$ 0.50$ per page Experimental Mathematics 12:4, page 507 


$$
\begin{gathered}
\left(\frac{1}{2}\right)_{n}\left(\frac{1}{2}\right)_{n}\left(\frac{1}{2}\right)_{n}\left(\frac{1}{4}\right)_{n}\left(\frac{3}{4}\right)_{n}, \\
\left(\frac{1}{2}\right)_{n}\left(\frac{1}{4}\right)_{n}\left(\frac{3}{4}\right)_{n}\left(\frac{1}{4}\right)_{n}\left(\frac{3}{4}\right)_{n}, \\
\left(\frac{1}{2}\right)_{n}\left(\frac{1}{3}\right)_{n}\left(\frac{2}{3}\right)_{n}\left(\frac{1}{3}\right)_{n}\left(\frac{2}{3}\right)_{n}, \\
\left(\frac{1}{2}\right)_{n}\left(\frac{1}{4}\right)_{n}\left(\frac{3}{4}\right)_{n}\left(\frac{1}{3}\right)_{n}\left(\frac{2}{3}\right)_{n}, \\
\left(\frac{1}{2}\right)_{n}\left(\frac{1}{4}\right)_{n}\left(\frac{3}{4}\right)_{n}\left(\frac{1}{6}\right)_{n}\left(\frac{5}{6}\right)_{n}, \\
\left(\frac{1}{2}\right)_{n}\left(\frac{1}{3}\right)_{n}\left(\frac{2}{3}\right)_{n}\left(\frac{1}{6}\right)_{n}\left(\frac{5}{6}\right)_{n}, \\
\left(\frac{1}{2}\right)_{n}\left(\frac{1}{6}\right)_{n}\left(\frac{5}{6}\right)_{n}\left(\frac{1}{6}\right)_{n}\left(\frac{5}{6}\right)_{n}, \\
\left(\frac{1}{2}\right)_{n}\left(\frac{1}{2}\right)_{n}\left(\frac{1}{2}\right)_{n}\left(\frac{1}{3}\right)_{n}\left(\frac{2}{3}\right)_{n}, \\
\left(\frac{1}{2}\right)_{n}\left(\frac{1}{2}\right)_{n}\left(\frac{1}{2}\right)_{n}\left(\frac{1}{6}\right)_{n}\left(\frac{5}{6}\right)_{n}, \\
\left(\frac{1}{2}\right)_{n}\left(\frac{1}{8}\right)_{n}\left(\frac{3}{8}\right)_{n}\left(\frac{5}{8}\right)_{n}\left(\frac{7}{8}\right)_{n}, \\
\left(\frac{1}{5}\right)_{n}\left(\frac{2}{5}\right)_{n}\left(\frac{3}{5}\right)_{n}\left(\frac{4}{5}\right)_{n}, \\
\left(\frac{1}{10}\right)_{n}\left(\frac{9}{10}\right)_{n},
\end{gathered}
$$

For $q$, we consider

$q=j^{2}, \quad q=j^{2}-1, \quad q=j^{3}, \quad q=\left(j^{2}-1\right)^{3}, \quad q=j^{4}$,

where $j$ is also an integer. We will look for integer relations between

$$
\begin{aligned}
& F_{0}=\sum_{n=0}^{\infty} \frac{B(n)}{q^{n}}, \quad F_{1}=\sum_{n=0}^{\infty} \frac{B(n)}{q^{n}} n, \\
& F_{2}=\sum_{n=0}^{\infty} \frac{B(n)}{q^{n}} n^{2}, \quad G=\frac{\sqrt{k}}{\pi^{2}} .
\end{aligned}
$$

This means that we want to find integers $a, b, c$, and $d$ such that $a F_{0}+b F_{1}+c F_{2}+d G=0, \quad d \neq 0$. The algorithms that solve this problem are called integer relations algorithms. The software we are using for this purpose is PARI-GP, because it is very fast at making numerical calculations and has the LINDEP function which looks for integer relations. To avoid the integer variable $k$, we also use a variant of this method and look for integer relations between

$$
F_{0}^{2}, \quad F_{1}^{2}, \quad F_{2}^{2}, \quad F_{0} F_{1}, \quad F_{0} F_{2}, \quad F_{1} F_{2}, \frac{1}{\pi^{4}} .
$$

This variant is especially interesting if there exist formulas with large values of $k$. The new formulas my computer found using these numerical methods are

$$
\begin{aligned}
& \sum_{n=0}^{\infty} \frac{(-1)^{n}\left(\frac{1}{2}\right)_{n}\left(\frac{1}{4}\right)_{n}\left(\frac{3}{4}\right)_{n}\left(\frac{1}{6}\right)_{n}\left(\frac{5}{6}\right)_{n}}{n !^{5} 2^{10 n}} \\
& \times\left(1640 n^{2}+278 n+15\right)=\frac{256 \sqrt{3}}{3 \pi^{2}}, \\
& \sum_{n=0}^{\infty} \frac{(-1)^{n}\left(\frac{1}{2}\right)_{n}\left(\frac{1}{4}\right)_{n}\left(\frac{3}{4}\right)_{n}\left(\frac{1}{3}\right)_{n}\left(\frac{2}{3}\right)_{n}}{n !^{5} 48^{n}} \\
& \times\left(252 n^{2}+63 n+5\right)=\frac{48}{\pi^{2}}, \\
& \sum_{n=0}^{\infty} \frac{(-1)^{n}\left(\frac{1}{2}\right)_{n}\left(\frac{1}{3}\right)_{n}\left(\frac{2}{3}\right)_{n}\left(\frac{1}{6}\right)_{n}\left(\frac{5}{6}\right)_{n}}{n !^{5} 80^{3 n}} \\
& \times\left(5418 n^{2}+693 n+29\right)=\frac{128 \sqrt{5}}{\pi^{2}}, \\
& \sum_{n=0}^{\infty} \frac{\left(\frac{1}{2}\right)_{n}\left(\frac{1}{8}\right)_{n}\left(\frac{3}{8}\right)_{n}\left(\frac{5}{8}\right)_{n}\left(\frac{7}{8}\right)_{n}}{n !^{5} 7^{4 n}} \\
& \times\left(1920 n^{2}+304 n+15\right)=\frac{56 \sqrt{7}}{\pi^{2}} .
\end{aligned}
$$

Once the software PARI-GP found these series, I used Maple to check again if they were correct. The numerical results show that they are correct to hundreds of digits. Now examine the following Ramanujan-type formulas [Borwein and Borwein 87, Chudnovsky and Chudnovsky 88, Ramanujan 14]:

$$
\begin{gathered}
\sum_{n=0}^{\infty} \frac{(-1)^{n}\left(\frac{1}{2}\right)_{n}\left(\frac{1}{4}\right)_{n}\left(\frac{3}{4}\right)_{n}}{n !^{3} 48^{n}}(28 n+3)=\frac{16 \sqrt{3}}{3 \pi} \\
\sum_{n=0}^{\infty} \frac{(-1)^{n}\left(\frac{1}{2}\right)_{n}\left(\frac{1}{6}\right)_{n}\left(\frac{5}{6}\right)_{n}}{n !^{3} 80^{3 n}}(5418 n+263)=\frac{640 \sqrt{15}}{3 \pi} \\
\sum_{n=0}^{\infty} \frac{\left(\frac{1}{2}\right)_{n}\left(\frac{1}{4}\right)_{n}\left(\frac{3}{4}\right)_{n}}{n !^{3} 7^{4 n}}(40 n+3)=\frac{49 \sqrt{3}}{9 \pi}
\end{gathered}
$$

It is interesting to observe that the numbers $48,80^{3}, 7^{4}$ are repeated in the denominators. This leads me to think that formulas of type (2-1), such as (1-1), (1-2), $(1-3),(2-2),(2-3),(2-4)$, and $(2-5)$, can be proved using the theory of modular functions, as is the case with Ramanujan-like formulas, (2-6), (2-7), and (2-8). 


\section{SUPPORTING THE CONJECTURE}

To support this conjecture, I will explain the origin of the number $80^{3}$ in formula $(2-7)$. We begin by considering Klein's absolute invariant [Borwein and Borwein 87, Chudnovsky and Chudnovsky 88]

$$
J(q)=\frac{4}{27} \frac{\left[1-\lambda(q)+\lambda^{2}(q)\right]^{3}}{\lambda^{2}(q)[1-\lambda(q)]^{2}}
$$

where

$$
\lambda(q)=\left[\frac{\vartheta_{2}(q)}{\vartheta_{3}(q)}\right]^{4} .
$$

It is known [Chudnovsky and Chudnovsky 88] that when $d$ is an integer such that $Q(\sqrt{-d})$ has class number 1 , then $J\left(\frac{1+\sqrt{-d}}{2}\right)$ is also an integer. For these singular values, there exist [Chudnovsky and Chudnovsky 88] integers $a, b, c, k$ such that

$$
\sum_{n=0}^{\infty} \frac{(12)^{3 n}\left(\frac{1}{2}\right)_{n}\left(\frac{1}{6}\right)_{n}\left(\frac{5}{6}\right)_{n}}{n !^{3} J^{n}\left(\frac{1+\sqrt{-d}}{2}\right)}(a n+b)=\frac{c \sqrt{k}}{\pi} .
$$

There are not many numbers with that property: 2, $3,7,11,19,43,67$, and 163 . For $d=43$, we have $J\left(\frac{1+\sqrt{-43}}{2}\right)=-960^{3}$, and the corresponding formula is $(2-7)$. Our new formula $(2-4)$ is intriguing because of the repetition of the numbers $80^{3}$ and 5418 . I think that one can find a proof of this formula using the theory of modular functions.

In [Berggren et al. 00], one can find the references [Chudnovsky and Chudnovsky 88] and [Ramanujan 14] and many more fascinating papers. In addition, the paper, [Berndt and Chan 01], reinforces the hope that the theory of modular forms is the key to proving the formulas developed in this paper.

\section{RELATED FORMULAS}

Boris Gourevitch [Gourevitch 02] has sent me, by email, the formula below for $1 / \pi^{3}$. He has found it by using integer relations algorithms:

$$
\sum_{n=0}^{\infty} \frac{\left(\frac{1}{2}\right)_{n}^{7}}{n !^{7} 2^{6 n}}\left(168 n^{3}+76 n^{2}+14 n+1\right)=\frac{32}{\pi^{3}} .
$$

On the other hand, we consider the functions

$$
F(k)=\sum_{n=0}^{\infty} \frac{(-1)^{n}}{2^{2 n}} \frac{\left(\frac{1}{2}+k\right)_{n}^{5}}{(1+k)_{n}^{5}}\left[20(n+k)^{2}+8(n+k)+1\right]
$$

$$
\begin{aligned}
& G(k)=\sum_{n=0}^{\infty} \frac{(-1)^{n}}{2^{10 n}} \frac{\left(\frac{1}{2}+k\right)_{n}^{5}}{(1+k)_{n}^{5}}\left[820(n+k)^{2}+180(n+k)+13\right] \\
& H(k)=\sum_{n=0}^{\infty} \frac{1}{2^{6 n}} \frac{\left(\frac{1}{2}+k\right)_{n}^{7}}{(1+k)_{n}^{7}}\left[168(n+k)^{3}+76(n+k)^{2}\right. \\
&\quad+14(n+k)+1] .
\end{aligned}
$$

We have seen in (1-3), (1-1), and (4-1) that

$$
F(0)=\frac{8}{\pi^{2}}, \quad G(0)=\frac{128}{\pi^{2}}, \quad H(0)=\frac{32}{\pi^{3}} .
$$

It is very curious that using the Simon Plouffe inverter [Plouffe], we find

$F\left(\frac{1}{2}\right)=7 \cdot \zeta(3), \quad G\left(\frac{1}{2}\right)=256 \cdot \zeta(3), \quad H\left(\frac{1}{2}\right)=\frac{\pi^{4}}{2}$.

The evaluation $G(1 / 2)$ has been proved by T. Amdeberhan [Amdeberhan 97].

\section{REFERENCES}

[Amdeberhan 97] T. Amdeberhan and D. Zeilberger. "Hypergeometric Series Acceleration via the WZ Method." Electronic J. Combinatorics 4:2. Available from World Wide Web (http://www. combinatoric.org/all_volumes.html), 1997.

[Berggren et al. 00] L. Berggren, J. Borwein, and P. Borwein. Pi: A Source Book. Berlin-Heidelberg: Springer-Verlag, 1997, 2000.

[Berndt and Chan 01] B. C. Berndt and H. H. Chan. "Eisenstein Series and Approximations to $\pi$." Illinois J. Math. 45 (2001), 75-90.

[Borwein and Borwein 87] J. Borwein and P. Borwein. Pi and the AGM. London: Wiley Interscience, 1987.

[Chudnovsky and Chudnovsky 88] D. V. Chudnovsky and G. V. Chudnovsky. Approximations and Complex Multiplication According to Ramanujan. San Diego, CA: Academic Press, 1988.

[Gourevitch 02] B. Gourevitch. Personal communication, 2002.

[Guillera 02] J. Guillera. "Some Binomial Series Obtained by the WZ-Method." Advances in Applied Mathematics 29 (2002), 599-603.

[Guillera 03] J. Guillera. "Generators of Some Ramanujan Formulas." To appear in The Ramanujan Journal.

[Plouffe] Simon Plouffe. "Plouffe's Inverter." Available from World Wide Web (http://pi.lacim.uqam.ca/eng/).

[Ramanujan 14] S. Ramanujan. "Modular Equations and Approximations to $\pi$." Quarterly Journal of Mathematics 45 (1914), 350-372. 
Jesús Guillera, Avda Cesáreo Alierta, 31 esc-izda, 4², Zaragoza-50008, Spain (jguillera@able.es)

Received April 22, 2003; accepted in revised form October 9, 2003. 